\title{
Genome-wide screening using array-CGH does not reveal microdeletions/microduplications in children with Kabuki syndrome
}

\author{
Jacqueline Schoumans ${ }^{*, 1}$, Ann Nordgren ${ }^{1,2}$, Claudia Ruivenkamp ${ }^{1,3}$, \\ Karen Brøndum- Nielsen ${ }^{4}$, Bin Tean Teh ${ }^{5}$, Göran Annéren ${ }^{6}$, Eva Holmberg ${ }^{7}$, \\ Magnus Nordenskjöld ${ }^{1}$ and Britt -Marie Anderlid ${ }^{1}$
}

\footnotetext{
${ }^{1}$ Department of Molecular Medicine, Karolinska Hospital, Stockholm, Sweden; ${ }^{2}$ Department of Clinical Genetics, Umea University Hospital, Sweden; ${ }^{3}$ Department of Human and Clinical Genetics, LUMC, Leiden, The Netherlands; ${ }^{4}$ John F Kennedy Institute, Glostrup, Denmark; ${ }^{5}$ Laboratory of Cancer Genetics, Van Andel Research Institute, Grand Rapids, MI, USA; ${ }^{6}$ Department of Clinical Genetics, Uppsala University Children's Hospital, Sweden; ${ }^{7}$ Department of Clinical Genetics, Sahlgrenska University Hospital/East, Gothenburg, Sweden
}

Kabuki syndrome (KS) is a rare multiple congenital anomaly/mental retardation syndrome. It is characterized by a distinct facial appearance, mental retardation, postnatal growth retardation, skeletal anomalies, unusual dermatoglyphics and fetal fingertip pads. It has previously been speculated that KS is caused by a microdeletion or duplication. In a recent report, an interstitial microduplication of 8p22-23.1 was presented in several cases with this disorder. We investigated 10 Caucasian patients diagnosed with $\mathrm{KS}$ by fluorescence in situ hybridization and microsatellite markers located on 8p22-23.1. Using the same clones that were previously reported to be duplicated on chromosome $8 p$, we could exclude the duplication in all our patients. In addition, we performed a genome-wide screening on this group of patients using array-based comparative genomic hybridization containing BAC clones spaced at approximately $1 \mathrm{Mb}$ intervals across the genome and could not find any evidence for gene dose alterations. The characteristics of KS are variable, a fact that complicates the diagnosis of this disorder. It is possible that we will find genetic heterogeneity among Kabuki patients, and therefore it is unlikely that all patients have an interstitial $8 p$ duplication. We conclude that the etiology of KS remains to be solved and further genetic studies are necessary to delineate its genetic cause.

European Journal of Human Genetics (2005) 13, 260-263. doi:10.1038/sj.ejhg.5201309

Published online 20 October 2004

Keywords: comparative genomic hybridization (CGH); microarray; microdeletion; Kabuki syndrome; duplication 8p; mental retardation

*Correspondence: J Schoumans, Department of Molecular Medicine, Karolinska Hospital, CMM L8:02, Stockholm SE-17176, Sweden.

Tel: + 468 51772521; Fax: + 468 51773620;

E-mail: Jacqueline.schoumans@cmm.ki.se

Received 21 May 2004; revised 25 August 2004; accepted 2 September 2004
Introduction

Kabuki syndrome (KS) was first independently described by Kuroki et al ${ }^{1}$ and Niikawa et al. ${ }^{2}$ It was initially believed to be fairly uncommon outside of Japan, but is now well recognized, and more than 350 cases have been reported worldwide. ${ }^{3,4}$ Although clinical manifestations of KS are well established, it is probably greatly underdiagnosed and the number of medical professionals who are familiar with the syndrome is still growing. Diagnosis is further 
complicated by the fact that the spectrum of symptoms is very diverse. Several chromosome abnormalities have been reported to be associated with KS. Matsumoto and Niikawa ${ }^{3}$ speculated on a possible microdeletion or microduplication as the cause of the disorder.

For successful detection of submicroscopic chromosome aberrations in patients with idiopathic mental retardation, ${ }^{5-10}$ different genome-wide high-resolution screening technologies have been applied during the past few years. Recently, Milunsky and Huang ${ }^{11}$ presented a 8 p22-23.1 duplication as a common etiologic basis for KS as a result of a screening of six patients using HR comparative genomic hybridization (HR-CGH). This finding prompted us to investigate $10 \mathrm{KS}$ patients for 8p22-23.1 duplications using fluorescence in situ hybridization (FISH) and microsatellite markers. In addition, we performed a whole genome screen on the same cases using array-based comparative genomic hybridization (array-CGH) in order to detect microdeletions or microduplications.

\section{Materials and methods \\ Patient selection}

Blood samples were collected from 10 patients who met cardinal clinical diagnostic criteria for KS. All patients were Caucasian of Swedish or Danish origin and all of them were evaluated by a clinical geneticist. Table 1 summarizes the major clinical findings.

\section{Cytogenetic analysis}

Metaphases from patient peripheral blood lymphocytes were prepared according to standard procedures. Routine chromosome analysis (450-500 bands) was performed in all cases without revealing any abnormalities.

\section{Array-CGH}

Genomic DNA was extracted from peripheral venous blood samples or cultured fibroblasts using Puregene blood kit (Gentra Systems Inc., Minneapolis, MN, USA) according to the manufacturer's protocol. Genomic DNA from the patients was digested into fragments of 100-2000 bp by overnight incubation with DpnII (New England Biolabs Inc., Beverly, MA, USA) at $37^{\circ} \mathrm{C}$ and checked on a $2 \%$ agarose gel. The DNA fragments were purified using Qiaquick PCR purification kit (Qiagen $\mathrm{GmbH}$, Hilden, Germany).

Array-CGH was performed using arrays containing 2600 BAC clones (Spectral Genomics Inc., Houston, TX, USA) according to the manufacturer's protocol with some modifications, previously described by Schoumans et al. ${ }^{9}$

\section{Microsatellite analysis}

Three microsatellite markers D8S550, D8S552 and D8S520 located on 8p22-23.1 were selected from Ensembl Genome Browser (http://ensembl.org, Build 34). ${ }^{12}$ Oligonucleotide primers labeled with NED (fluorescent dye) were obtained from Applied Biosystems. Polymerase chain reaction (PCR) amplification of genomic DNA from patients $(50 \mathrm{ng}$ ) was performed according to the manufacturer's protocol and analyzed using an automated sequencer (ABI PRISM ${ }^{\circledR} 3100$ Avant Genetic analyzer, Applied Biosystems, Foster City, CA, USA). Resulting genotype data were analyzed using Genemapper ${ }^{\circledR} 3.5$ software from ABI (Applied Biosystems). Alleles from patients and their

Table 1 Clinical findings

\begin{tabular}{|c|c|c|c|c|c|c|c|c|c|c|}
\hline Case & 1 & 2 & 3 & 4 & 5 & 6 & 7 & 8 & 9 & 10 \\
\hline Age (years) & 4 & 15 & 6 & 14 & 7 & 2 & 5 & 8 & 9 & 7 \\
\hline Gender & $\mathrm{F}$ & $\mathrm{M}$ & $\mathrm{F}$ & $\mathrm{M}$ & $\mathrm{F}$ & $\mathrm{F}$ & $\mathrm{F}$ & $\mathrm{F}$ & $\mathrm{F}$ & $\mathrm{F}$ \\
\hline Prenatal growth retard. & + & - & - & + & - & - & - & + & + & NA \\
\hline Postnatal growth retard. & + & + & + & + & - & - & - & + & $(+)$ & $(+)$ \\
\hline Mental retardation & MMR & ModMR & $\mathrm{MMR} / \mathrm{N}$ & ModMR & SMR & SMR & SMR & MMR & MḾR & ModMR \\
\hline Seizures & $+^{*}$ & + & - & + & + & + & + & - & + & - \\
\hline Hypotonia & + & + & + & - & + & + & + & $(+)$ & + & + \\
\hline Long palpebral fissures & + & + & + & + & + & + & + & + & + & + \\
\hline Eversion of lower eyelid & + & + & + & - & + & + & + & + & + & + \\
\hline Arched eyebrows & + & + & + & + & + & + & - & + & + & + \\
\hline Large ears & + & + & + & + & + & + & + & + & + & + \\
\hline Depressed nasal tip & + & + & + & + & + & + & + & $(+)$ & + & - \\
\hline Abnormal dentition & - & + & + & + & + & NA & - & + & NA & + \\
\hline Skeletal abnormalities & + & - & + & + & - & - & - & + & NA & NA \\
\hline Brachydactyly V & + & + & + & + & + & NA & NA & - & - & NA \\
\hline Fetal finger pads & + & + & + & + & + & + & + & NA & + & + \\
\hline Congenital heart defect & + & - & - & - & - & - & - & - & + & - \\
\hline Lip pits & - & - & - & - & + & - & - & - & - & - \\
\hline Renal abnormality & - & - & + & + & - & $\mathrm{NI}$ & $\mathrm{NI}$ & - & - & - \\
\hline
\end{tabular}

ModMR = moderate $M R ; M M R=$ mild $M R ; S M R=$ severe $M R ; N=$ normal; $F=$ female; $M=m a l e ; N I=$ not investigated; (+)=slightly; ${ }^{*}$ febrile convulsion; $\mathrm{NA}=$ data not available. 
parents were scored, and the microsatellite allele signal intensities were in addition compared to normal DNA controls.

\section{FISH analysis}

The following clones located on 8p22-23.1 were used for FISH analysis: RP11-92C1, RP11-31B7 and RP11-112G9. Clone RP11-23H1 was used as control. For investigation of polymorphic clones, RP11-259N12, RP11-316O14, RP11100C24 and RP11-125A5 were used. All clones were obtained from The Welcome Trust Sanger Institute (Cambridge, UK). Bacterial cultures and DNA isolation were performed according to the BAC-PAC miniprep protocol (http://www.biologia.uniba.it/rmc). Probes were labeled with FITC-dUTP (NEN Life Science Products, Boston, MA, USA) or SpectrumOrange-dUTP (Vysis Inc, Downers Grove, IL, USA) by nick translation, and FISH analyses were performed according to a standard protocol on metaphase slides that were prepared from lymphocyte cultures of peripheral blood. The slides were analyzed on a Zeiss Axioplan 2 (Carl Zeiss, Göttingen, Germany) epifluorescence microscope and images were captured using a cooled CCD camera (Sensys Photometrics, München, Germany) and SmartCapture 2 software (DigitalScientific Ltd, Cambridge, UK). FISH signals were examined on both metaphase chromosomes and interphase nuclei. Inverted DAPI staining was used for chromosome identification during FISH analysis.

\section{Results}

The results of all experiments are summarized in Table 2 .

We investigated in detail chromosome region $8 \mathrm{p} 22-23.1$ in all 10 patients diagnosed with KS using the same clones reported by Milunsky and Huang. ${ }^{11}$ We performed metaphase and interphase FISH analysis on five patients (case 1, 2 and 4-6). In the other five cases (case 3 and 7-10), cells were not available for FISH analysis and we therefore examined these patients using three microsatellite markers located in the same region. None of the clones or microsatellite markers revealed a duplication in our KS patients.

In addition, we used a whole genome array containing 2600 clones with an average resolution of $1 \mathrm{Mb}$ to screen the patients for microdeletions or microduplications. Among these, three clones were covering $>3 \mathrm{Mb}$ fragment at 8p22-8p23.1 (RP11-252K12, RP11-80B8 and RP11$90017)$. None of these clones revealed a duplication in any of the patients investigated, nor did we observe any other chromosomal imbalances.

However, in some patients we observed gains or losses on four BAC clones (listed in Table 3).

\section{Discussion}

We have studied 10 cases with KS for gene dose alterations. We were unable to repeat the observation of chromosome band 8p22-23.1 duplications with FISH or microsatellite analysis in our patients. Furthermore, array-CGH analysis with 2600 clones evenly distributed over the human genome with $1 \mathrm{Mb}$ intervals did not reveal any alterations, except for four polymorphic clones. Variations in the genetic material among phenotypically normal individuals have been known to occur in the form of single base pair changes. However, very recently Iafrate et $a l^{13}$ reported large-scale copy-number variations involving gains or losses of several kilobases to hundreds of kilobases of genomic DNA. One of the polymorphic clones observed in our study (RP11-259N12) was also reported by Iafrate et al

Table 3 Polymorphic clones

\begin{tabular}{llccc}
\hline Clone ID & Cytogenetic location & Gain & Loss & Case \\
\hline RP11-259N12 & $1 \mathrm{p} 21.1$ & 1 & 2 & $1,3,8$ \\
RP11-316O14 & $2 \mathrm{q} 35$ & 4 & 0 & $3,4,6,7$ \\
RP11-100C24 & $13 q 21.1$ & 2 & 0 & 3,8 \\
RP11-125A5 & $14 q 12$ & 0 & 3 & $1,4,6$ \\
\hline
\end{tabular}

Table 2 Summary of gene dose analysis

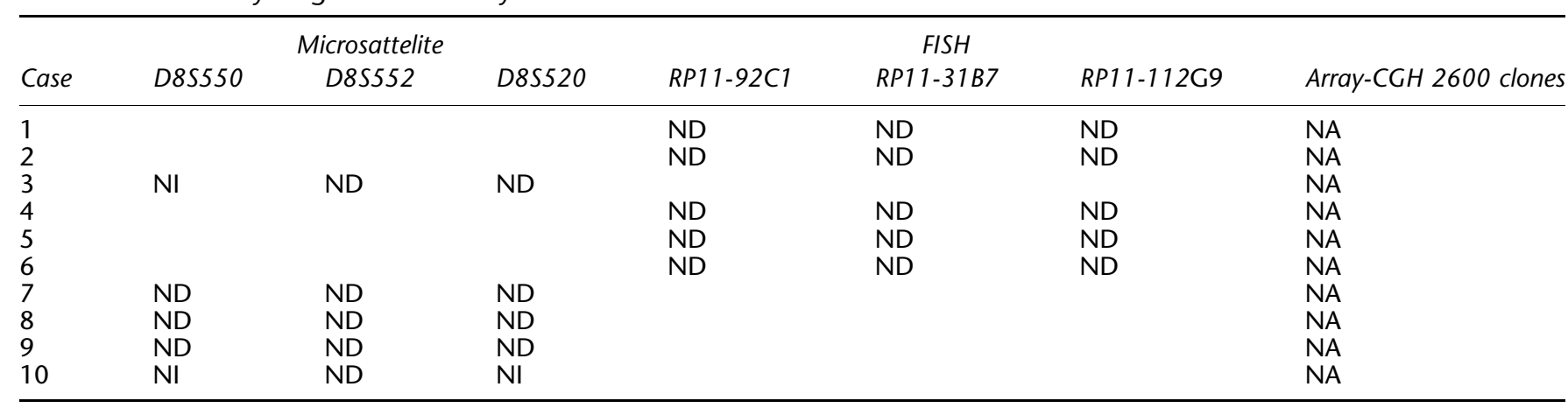

$\mathrm{NA}=$ no abnormality; $\mathrm{ND}=$ no duplication; $\mathrm{NI}=$ not informative. 
and was only confirmable by FIBER-FISH analysis. We experienced the same difficulties as Iafrate et al to confirm gains or losses in our patients by conventional metaphaseand interphase-FISH analysis using the four clones listed in Table 3. However, the same clones are listed in the public accessible Genome Variation Database at http://projects. tcag.ca/variation, since they are frequently polymorphic in healthy individuals and they are therefore not considered to contribute to the cause of KS.

Taken together, our results suggest that gene dose alterations in $8 \mathrm{p}$ are unlikely to be the cause in our series of KS cases. Miyake et al, ${ }^{14}$ who performed a thorough investigation of region 8 p22-23.1 in $26 \mathrm{KS}$ patients, came to the same conclusion.

The previously reported $8 \mathrm{p}$ duplication in six KS patients was relatively large in size $(3.5 \mathrm{Mb})$, which should be easily detected. Our inability to detect the duplication might have different explanations. Firstly, the $8 \mathrm{p}$ region is prone to genetic rearrangements due to large olfactory receptor (OR)-gene clusters causing unequal crossovers between two OR clusters in this region. ${ }^{15}$ Imbalances of the $8 p$ region have been frequently reported with a wide range of different phenotypes, and also in individuals without clinical manifestations. ${ }^{16,17}$ The duplication reported by Milunsky and Huang could thus be a normal variant present in their patient population.

Secondly, the wide spectrum of characteristics of KS complicates the diagnosis and it is therefore likely that KS is clinically and genetically heterogeneous.

In conclusion, our data demonstrate that the etiology of KS remains unknown. It may be worthwhile to further investigate this group of patients using a microarray covering the whole genome at a much higher resolution. ${ }^{18}$

\section{Acknowledgements}

We are greatly indebted to the patients and their parents. We also thank our Clinical colleagues from Swedish and Danish pediatric departments for referring the patients and the Welcome Trust Sanger Institute, UK for providing the BAC clones. This work was supported by funds from the Swedish Medical Research Council, Frimurarna Barnahuset Foundation, Linnea och Josef Carlsson Stiftelse, Stiftelsen Sävstaholm and the Ronald McDonald Child Foundation.

\section{References}

1 Kuroki Y, Suzuki Y, Chyo H, Hata A, Matsui I: A new malformation syndrome of long palpebral fissures, large ears, depressed nasal tip, and skeletal anomalies associated with postnatal dwarfism and mental retardation. J Pediatr 1981; 99: 570-573.

2 Niikawa N, Matsuura N, Fukushima Y, Ohsawa T, Kajii T: Kabuki make-up syndrome: a syndrome of mental retardation, unusual facies, large and protruding ears, and postnatal growth deficiency. J Pediatr 1981; 99: 565-569.

3 Matsumoto N, Niikawa N: Kabuki make-up syndrome: a review. Am J Med Genet 2003; 117C: 57-65.

4 Wessels MW, Brooks AS, Hoogeboom J, Niermeijer MF, Willems PJ: Kabuki syndrome: a review study of three hundred patients. Clin Dysmorphol 2002; 11: 95-102.

5 Kirchhoff M, Rose H, Lundsteen C: High resolution comparative genomic hybridisation in clinical cytogenetics. J Med Genet 2001; 38: $740-744$.

6 Ness GO, Lybaek H, Houge G: Usefulness of high-resolution comparative genomic hybridization (CGH) for detecting and characterizing constitutional chromosome abnormalities. Am J Med Genet 2002; 113: 125-136.

7 Shaw-Smith C, Redon R, Rickman L et al: Microarray based comparative genomic hybridisation (array-CGH) detects submicroscopic chromosomal deletions and duplications in patients with learning disability/mental retardation and dysmorphic features. J Med Genet 2004; 41: 241-248.

8 Vissers LE, de Vries BB, Osoegawa K et al: Array-based comparative genomic hybridization for the genomewide detection of submicroscopic chromosomal abnormalities. Am J Hum Genet 2003; 73: $1261-1270$.

9 Schoumans J, Anderlid BM, Blennow E, Teh BT, Nordenskjold M: The performance of CGH array for the detection of cryptic constitutional chromosome imbalances. J Med Genet 2004; 41: 198-202.

10 Schoumans J, Nielsen K, Jeppesen I et al: A comparison of different metaphase CGH methods for the detection of cryptic chromosome aberrations of defined size. Eur J Hum Genet 2004; 12: $447-454$.

11 Milunsky JM, Huang XL: Unmasking Kabuki syndrome: chromosome 8 p22-8p23.1 duplication revealed by comparative genomic hybridization and BAC-FISH. Clin Genet 2003; 64: 509-516.

12 Birney E, Andrews D, Bevan P et al: Ensembl 2004. Nucleic Acids Res 2004; 32 (Database issue): D468-470.

13 Iafrate AJ, Feuk L, Rivera MN et al: Detection of large-scale variation in the human genome. Nat Genet 2004; 36: 949-951.

14 Miyake N, Harada N, Shimokawa O et al: On the reported 8p22-p23.1 duplication in Kabuki make-up syndrome (KMS) and its absence in patients with typical KMS. Am J Med Genet 2004; 128A: $170-172$.

15 Giglio S, Broman KW, Matsumoto N et al: Olfactory receptor-gene clusters, genomic-inversion polymorphisms, and common chromosome rearrangements. Am J Hum Genet 2001; 68: 874-883.

16 Engelen JJ, Moog U, Evers JL, Dassen H, Albrechts JC, Hamers AJ: Duplication of chromosome region 8 p23.1 $->$ p23.3: a benign variant? Am J Med Genet 2000; 91: 18-21.

17 Tsai CH, Graw SL, McGavran L: 8p23 duplication reconsidered: is it a true euchromatic variant with no clinical manifestation? J Med Genet 2002; 39: 769-774.

18 Ishkanian AS, Malloff CA, Watson SK et al: A tiling resolution DNA microarray with complete coverage of the human genome. Nat Genet 2004; 36: 299-303. 